\title{
Enzymatic emulsifier-free emulsion polymerization to prepare polystyrene particles using horseradish peroxidase as a catalyst
}

\author{
Michinari Kohri, Satoshi Uzawa, Ayaka Kobayashi, Haruka Fukushima, Tatsuo Taniguchi \\ and Takayuki Nakahira
}

Polymer Journal (2013) 45, 354-358; doi:10.1038/pj.2012.129; published online 27 June 2012

Keywords: emulsifier-free emulsion polymerization; enzymatic polymerization; horseradish peroxidase; polymer particle

\section{INTRODUCTION}

Polymer particles are widely used in many areas, such as the technical and biomedical fields. ${ }^{1}$ The size and surface characteristics are extremely important factors that influence the properties of polymer particles. Various methods have been developed for synthesizing polymer particles. ${ }^{2,3}$ Among the available synthesis methods, emulsifier-free emulsion polymerization is particularly useful for preparing polymer particles that have a narrow particlesize distribution and a well-characterized surface in the absence of surfactants. ${ }^{4}$ Interestingly, surfactant-free latexes are easy to purify. The colloidal stabilities of the obtained particles are enhanced by the following components, which act like emulsifiers: an ionizable initiator (for example, potassium persulfate) and hydrophilic comonomers (for example, sodium styrene sulfonate).

Enzymes are useful catalysts for the syntheses of polymeric materials. ${ }^{5-7}$ Among these enzymes, horseradish peroxidase (HRP) has been used for the oxidative polymerization of aromatic compounds in the presence of $\mathrm{H}_{2} \mathrm{O}_{2} 8,9$ and the free-radical polymerization of vinyl monomers in the presence of $\mathrm{H}_{2} \mathrm{O}_{2}$ and $\beta$-diketones. ${ }^{10,11}$ Most researches that have used HRP as a catalyst have focused on solution and bulk polymerization. However, some studies have examined the synthesis of polymer particles using HRP as the catalyst. The HRP-catalyzed dispersion polymerization of phenol derivatives ${ }^{12,13}$ or aniline derivatives ${ }^{14}$ and an emulsion polymerization of vinyl monomers ${ }^{15}$ have been reported. Schork et al..$^{16}$ and our group ${ }^{17}$ have separately investigated HRP-mediated miniemulsion polymerizations for preparing polystyrene particles that have diameters of approximately $50 \mathrm{~nm}$. Furthermore, we have reported the use of particles containing $\beta$-diketone moieties as substrates for a surface-initiated HRP-mediated polymerization. ${ }^{18}$

Although extensive work has been reported on emulsifier-free emulsion polymerization using various types of initiators, there are no literature reports describing the use of an enzymatic reaction as the initiator. For this study, polystyrene particles were prepared via the HRP-mediated emulsifier-free emulsion polymerization of styrene (Figure 1). The obtained particles were characterized using dynamic light scattering, scanning electron microscopy (SEM) and gel permeation chromatography. A mechanism for the formation of the particles is proposed. A comparison of three $\beta$-diketones (that is, acetylacetone (ACAC), 1,3-cyclopentanedione $(\mathrm{CP})$ and benzoylacetone $(\mathrm{BA})$ ) was conducted for the enzymatic emulsifier-free emulsion polymerizations. The results indicate that enzymatic emulsifier-free emulsion polymerization has a great potential to be used as an environmentally friendly synthetic process for the formation of polymer particles, which provides a promising avenue for green chemistry progress.

\section{EXPERIMENTAL PROCEDURES}

\section{Materials}

Styrene, $\mathrm{H}_{2} \mathrm{O}_{2}(30 \mathrm{wt} \%)$ and ACAC were purchased from the Kanto Chemical Co., Inc. (Tokyo, Japan). HRP $\left(383 \mathrm{U} \mathrm{mg}^{-1}\right.$, molecular weight $\left.=40000\right)$, CP and BA were purchased from Wako Pure Chemical Industries Ltd., Osaka, Japan. The styrene was dried over calcium hydride and distilled under reduced pressure before use. All other chemicals and solvents were of reagent grade and used as received.

\section{Measurements}

The hydrodynamic diameters $\left(D_{\mathrm{h}}\right)$ of the particles in water were measured using dynamic light scattering (Zetasizer Nano series Nano-ZS; Malvern Instruments, Ltd., Malvern, UK). Gel permeation chromatography was conducted (TSKgel $\alpha$ 3000; Tosoh Corp., Tokyo, Japan) using tetrahydrofuran as the mobile phase at a flow rate of $0.4 \mathrm{ml} \mathrm{min}^{-1}$ at $40{ }^{\circ} \mathrm{C}$. Polystyrene standards with a polydispersity $\left(M_{\mathrm{w}} / M_{\mathrm{n}}\right)<1.2$ were used to calibrate the gel permeation chromatography. The SEM micrographs were acquired using a scanning electron microscope (JSM6510A; JEOL Ltd., Tokyo, Japan) operating at $15-20 \mathrm{kV}$. For each sample, the sizes of 100 particles were measured to calculate the size (that is, diameter), size distribution and coefficient of variation. 


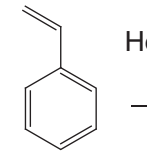

styrene
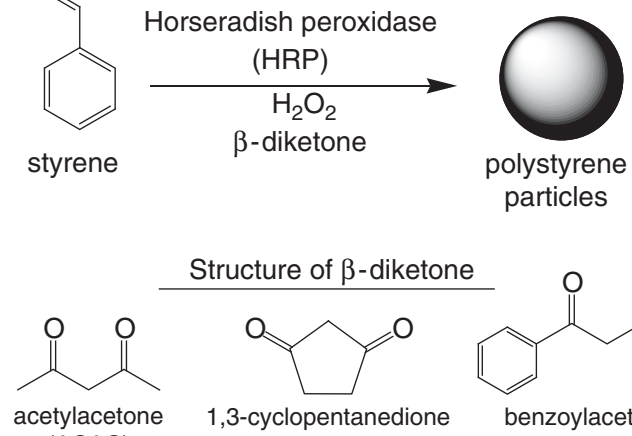

(ACAC)

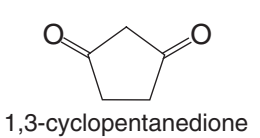

Structure of $\beta$-diketone

(CP)
(BA)

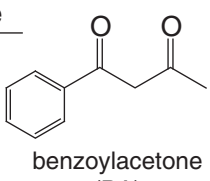

Figure 1 The general reaction scheme for the enzymatic emulsifier-free emulsion polymerization of styrene and the structures of the $\beta$-diketones.

\section{Enzymatic emulsifier-free emulsion polymerization of styrene}

A mixture of styrene $(110 \mu \mathrm{l}, 0.1 \mathrm{mmol})$ and deionized water $(3.0 \mathrm{ml})$ was placed into a three-necked flask. The mixture was deoxygenated using an argon purge for $15 \mathrm{~min}$. HRP ( $6.8 \mathrm{mg}, 2600 \mathrm{U}$ ) was dissolved in a small amount of deionized water and purged with nitrogen for $10 \mathrm{~min}$. Some of the HRP solution was injected into the mixture at room temperature. Subsequently, $\mathrm{H}_{2} \mathrm{O}_{2}(1 \mu \mathrm{l}, 10 \mu \mathrm{mol})$ and a $\beta$-diketone (that is, ACAC, CP or BA $(3 \mu \mathrm{l}$, $3 \mu \mathrm{mol})$, dissolved in styrene monomer) were added. The polymerization solution was stirred for $6 \mathrm{~h}$ under nitrogen, and aliquots were withdrawn periodically. The monomer conversion was determined by freeze drying and then weighing aliquots that were periodically withdrawn from the polymerization medium. Before the measurements, the obtained particles were separated and purified repeatedly by centrifugation (13500 r.p.m., $15 \mathrm{~min}$ ) and redispersion.

\section{RESULTS AND DISCUSSION}

The styrene conversion versus time curves are shown in Figure 2a. For these experiments, ACAC was used as the $\beta$-diketone. During the initial stage of polymerization, the conversion of styrene proceeded slowly until $60 \mathrm{~min}$. This phenomenon is likely attributable to the low initiation rate of the polymerization. $\mathrm{H}_{2} \mathrm{O}_{2}$ was added every $15 \mathrm{~min}$ (for a total of five times) with vigorous stirring to avoid the inactive form of HRP (Eiii), which forms under excess $\mathrm{H}_{2} \mathrm{O}_{2} \cdot{ }^{19,20}$ Adding $\mathrm{H}_{2} \mathrm{O}_{2}$ to the reaction mixture all at once caused a significant amount of the monomer to remain in the reaction mixture, which resulted in a lower conversion rate of the polymers. The conversion significantly increased as the reaction time increased, and then reached a plateau after approximately $180 \mathrm{~min}$. This result indicates that a second radical caused an instantaneous bimolecular termination, which was predicted in the emulsion polymerization mechanism described by Smith and Ewart.

Previous reports have explained the importance of examining the influence of the $\mathrm{H}_{2} \mathrm{O}_{2}$ to $\mathrm{HRP}$ initial concentration ratio $(\alpha){ }^{17,19}$ Therefore, several experiments with an $\alpha$ of 60-900 were prepared. The effects of $\alpha$ on the conversion of styrene are presented in Figure $2 \mathrm{~b}$. At low $\alpha(\alpha=60)$, the initiation rate was too low to form polymers. Increasing $\alpha$ to $190-600$ caused an increase in the amount of conversion to approximately $30 \%$. The results indicated that an upper limit exists for $\alpha$; for $\alpha$ values $>900$, the styrene conversion gradually diminished after $24 \mathrm{~h}$. Recently, we reported the preparation of polystyrene particles using HRP-mediated miniemulsion polymerization (vide supra). ${ }^{17}$ For that enzymatic miniemulsion polymerization, we did not observe an $\alpha$ upper limit. Even at higher $\alpha$ (approximately 23000), the conversion was maintained at approximately $100 \%$. These differences are likely attributable to
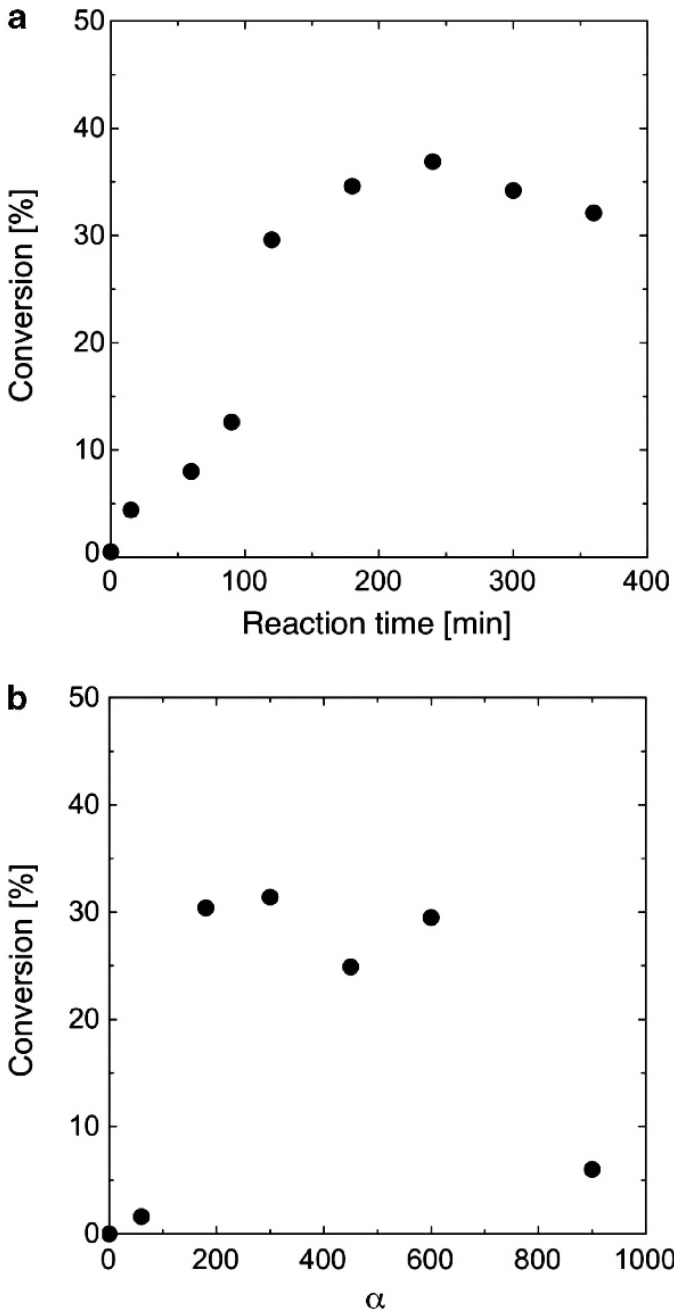

Figure 2 (a) Effects of reaction time on styrene conversion of the HRPmediated emulsifier-free emulsion polymerization. The polymerization was conducted at room temperature with ACAC ( $3 \mu \mathrm{mol})$. (b) Effects of $\alpha$ on the styrene conversion of the HRP-mediated emulsifier-free emulsion polymerizations. Polymerizations were conducted at room temperature for $6 \mathrm{~h} .(\mathrm{ACAC}=3 \mu \mathrm{mol})$.

the particle-formation mechanisms. The proposed mechanisms of enzymatic miniemulsion and emulsifier-free emulsion polymerization are schematically presented in Figure 3. In both cases, during the initial stage of enzymatic polymerization, ACAC radicals are generated by the HRP-mediated oxidation in the aqueous phase, and the water-soluble monomers might have initially polymerized. As shown in Figure 3a, the growing chain molecules in the enzymatic miniemulsion polymerizations become increasingly less water-soluble; this process results in rapid absorption into the monomer droplets that are stabilized with surfactants. These conditions result in the formation of polymer particles. Consequently, the HRP and styrene monomers are almost perfectly separated. However, the particle nucleation resulting from the enzymatic emulsifier-free emulsion polymerization is divisible into two stages, as shown in Figure $3 \mathrm{~b}$. First, a small amount of the monomer molecules, which are dissolved in the aqueous phase, are initiated by the ACAC radicals and polymerized as described above. During this stage, the monomer conversion is usually low, and most of the monomers are located in 

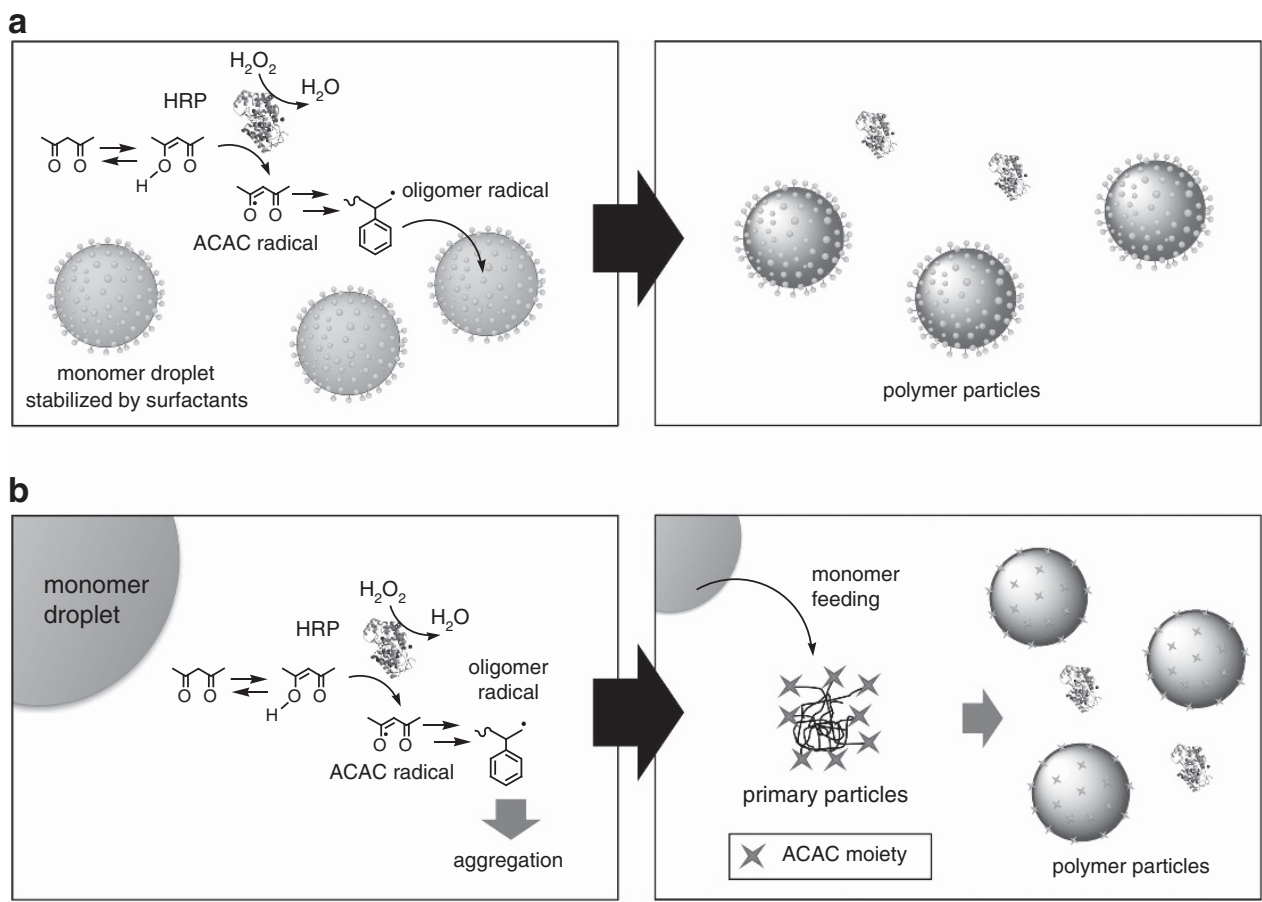

Figure 3 Proposed mechanisms of (a) enzymatic miniemulsion polymerization and (b) enzymatic emulsifier-free emulsion polymerization using the HRP/ $\mathrm{H}_{2} \mathrm{O}_{2} /$ ACAC system.

large monomer droplets $(1-10 \mu \mathrm{m}$ in diameter). Oligomer radicals that formed in the aqueous phase grow via precipitation and aggregation, which leads to the formation of primary particles. Polymerization of the monomers continues within the swollen primary particles, which are supplied by diffusion from the monomer droplets. The resultant polymer particles are stabilized by ACAC moieties, which are located on the surface of the particles. Although ketones do not have a charge, the hydrophilic character of ACAC provides stabilization through steric interactions of the formed particles. Pich et al. $^{21}$ reported a similar observation for the preparation of polystyrene particles functionalized with $\beta$-diketone groups via the emulsifier-free emulsion co-polymerization of styrene and acetoacetoxyethyl methacrylate. Furthermore, some HRPs apparently adsorbed onto the surface of the particles and acted as a steric stabilizer, which enhanced the colloidal stability of the resultant particles. In this mechanism, HRP is continually in contact with the styrene monomer, which results in the partial degradation of the HRP. The degradation of HRP leads to an increased probability that the inactive form of HRP (Eiii) will form under excess $\mathrm{H}_{2} \mathrm{O}_{2} \cdot{ }^{19,20}$ Therefore, during enzymatic emulsifier-free emulsion polymerization, an upper limit of $\alpha$ is observed.

The $\beta$-diketone structure was shown to affect the properties of the resultant polymer. Lalot et al., ${ }^{19}$ Kaplan et al. ${ }^{20}$ and our group ${ }^{17}$ have each reported that the structure of the $\beta$-diketone influences HRPmediated polymerization. Therefore, three $\beta$-diketones were selected and used as initiators for the polymerization reaction to investigate the effects of the $\beta$-diketone structures on the enzymatic emulsifierfree emulsion polymerization. Using BA (an oil-soluble $\beta$-diketone) as an initiator caused most of the styrenes to remain unpolymerized. During HRP-mediated polymerizations of vinyl monomers, $\mathrm{H}_{2} \mathrm{O}_{2}$ oxidizes the HRP, and then the oxidized metal center is reduced by the $\beta$-diketone, which produces $\beta$-diketone-derived radicals in the aqueous phase. Therefore, BA molecules that are incorporated inside

\section{Table 1 Characterization of particles ${ }^{\mathrm{a}}$}

\begin{tabular}{lccccc}
\hline Entry & $\beta$-diketone $(\mu \mathrm{mol})$ & Conversion (\%) & $\mathrm{M}_{n}{ }^{\mathrm{b}}$ & $\mathrm{M}_{w} \mathrm{M}_{n}{ }^{\mathrm{b}}$ & $\mathrm{D}_{h}{ }^{\mathrm{c}}(\mathrm{nm})$ \\
\hline 1 & ACAC: 0 & $\mathrm{ND}$ & $\mathrm{ND}$ & $\mathrm{ND}$ & $\mathrm{ND}$ \\
2 & ACAC: 5 & 23.4 & 37800 & 3.4 & 152.7 \\
3 & ACAC: 15 & 51.1 & 18400 & 2.7 & 157.6 \\
4 & ACAC: 20 & 40.0 & 10300 & 2.4 & 144.3 \\
5 & CP: 0 & $\mathrm{ND}$ & $\mathrm{ND}$ & $\mathrm{ND}$ & $\mathrm{ND}$ \\
6 & $\mathrm{CP}: 5$ & 26.2 & 21800 & 4.1 & 308.9 and 1235 \\
7 & CP: 15 & 32.5 & 17200 & 2.9 & 268.9 and 1179 \\
8 & CP: 20 & 39.0 & 21700 & 3.7 & 287.8 and 1421 \\
\hline
\end{tabular}

Abbreviations: ACAC, acetylacetone; CP, 1,3-cyclopentanedione; ND, not detected. apolymerization was conducted at room temperature for $6 \mathrm{~h}$.

bValues obtained from gel permeation chromatography (GPC) measurements.

'Values obtained from dynamic light scattering (DLS) measurements. The corresponding DLS data are shown in Supplementary Figure S1.

the styrene monomer droplets are unable to contribute to the radical initiation because they react with the water-soluble HRP and $\mathrm{H}_{2} \mathrm{O}_{2}$. In contrast, $\mathrm{ACAC}$ and $\mathrm{CP}$ (that is, water-soluble $\beta$-diketones) generated polymer particles at a conversion rate of approximately $25-50 \%$ (Table 1). The initial amount of $\beta$-diketone influenced the molecular weight of the resultant polymer because the $\beta$-diketone radicals generated during the HRP-catalyzed oxidation of $\beta$-diketone initiate the polymerization reaction (vide supra). To investigate this effect, the molecular weights of the obtained particles that were formed using various amounts of ACAC were measured using gel permeation chromatography. The $M_{\mathrm{n}}$ decreased from approximately 37800 to approximately 10300 , as the amount of ACAC increased, which clearly indicates that the amount of ACAC influences the molecular weight of the obtained particles (Table 1, entries 2-4). The polydispersity $\left(M_{\mathrm{w}} / M_{\mathrm{n}}\right)$ of the polymer generated from ACAC was 
approximately 2.4-3.4. However, relatively similar values of $M_{\mathrm{n}}$ (20000) were obtained for the polymers generated when CP was used as the $\beta$-diketone (Table 1 , entries $6-8$ ). This phenomenon is not yet fully understood, and additional experiments are necessary for clarification. Although polymers prepared by emulsion polymerization typically have higher molecular weights compared with polymers prepared using other polymerization methods, the polystyrene obtained from the conditions used in this study had lower molecular weights. The reason for the low molecular weight was likely because of the large amount of $\beta$-diketones used. When a low amount of $\beta$ diketone (for example, 0.1-1 $\mu \mathrm{mol}$, which was $0.1-1 \mathrm{~mol} \%$ with respect to the styrene monomer) was used, the styrene conversion was nearly $0 \%$, which indicates that the initiation rate was too low to enable significant polymerization. Therefore, the enzymatic polymerization reaction was performed using a $\beta$-diketone amount between 5 and $15 \mu \mathrm{mol}$ (5-15 mol\% with respect to styrene monomer), which is high compared with conventional polymerization conditions.

The effects of the $\beta$-diketone structure (ACAC or $\mathrm{CP}$ ) on the formation of the particles were investigated using SEM Figure $4 \mathrm{~A}$ presents the SEM micrographs of the obtained particles (Table 1, entries 3 and 6). When ACAC was used as an initiator, spherical and monodisperse polystyrene particles were obtained with a diameter distribution (that is, coefficient of variation) of approximately $5.6 \%$, as shown in Figure $4 \mathrm{~A}$ (a). The obtained polymer particles had diameters that were approximately $158 \mathrm{~nm}$, which is highly consistent with the results obtained from the dynamic light scattering measurements (Supplementary Figure S1). Furthermore, a dispersed solution of the particles was very stable; no sign of aggregated precipitation was observed over a 1-month duration (Figure 4B (a)). No surfactants or comonomers were used to stabilize the particles. Therefore, the stability of the dispersion primarily resulted from the interparticle repulsion caused by the ACAC moieties located on the surface of the particles. In contrast, when CP was used as the initiator, the obtained particles were polydisperse and the average diameter was approximately $203 \mathrm{~nm}$ (coefficient of variation=approximately 18.2\%), as shown in Figure 4A (b). Although the resultant particles were dispersed in water, the particles partially aggregated and settled to the bottom of the sample vial after 1 month (Figure 4B (b)). When the diameters of the particles in water were measured using dynamic light scattering, two peaks, at approximately 287 and $1421 \mathrm{~nm}$, were observed, which were attributed to the individual particles and aggregates, respectively Supplementary Figure S1. These results indicate that the particles with $\mathrm{CP}$ moieties on their surface had lower dispersion stability than the particles with ACAC moieties. Therefore, the presence of the $\beta$-diketone moieties on the surface of the particles apparently affected the colloidal stability of the resultant particles.

The colloidal stability of the nuclei decreased with an increasing rate of nuclei generation and radical concentration because the steric repulsion was not controlled. Therefore, the final size of the particles increased as the radical concentration increased. In the present system, $\beta$-diketone radicals were generated from the HRP-mediated reactions. The enol form is generally assumed to represent the actual substrate for the enzymatic radical formation reaction, and the state of keto-enol equilibrium determines the actual substrate concentration. ${ }^{17,22}$ When the enol content in the water of ACAC is approximately $16 \%$, the content of CP is approximately $100 \%{ }^{17}$ Therefore, the difference in the particle diameters and distributions likely results from the ratio of the keto-enol tautomers.

\section{CONCLUSIONS}

Polystyrene particles were prepared using the HRP-mediated emulsifier-free emulsion polymerization of styrene using $\mathrm{ACAC}$ or $\mathrm{CP}$ as the initiator in water at room temperature. The $\mathrm{H}_{2} \mathrm{O}_{2}$ to HRP initial concentration ratio $(\alpha)$ was important for the optimization of the reaction. The results indicate that the $\beta$-diketone moieties, which were
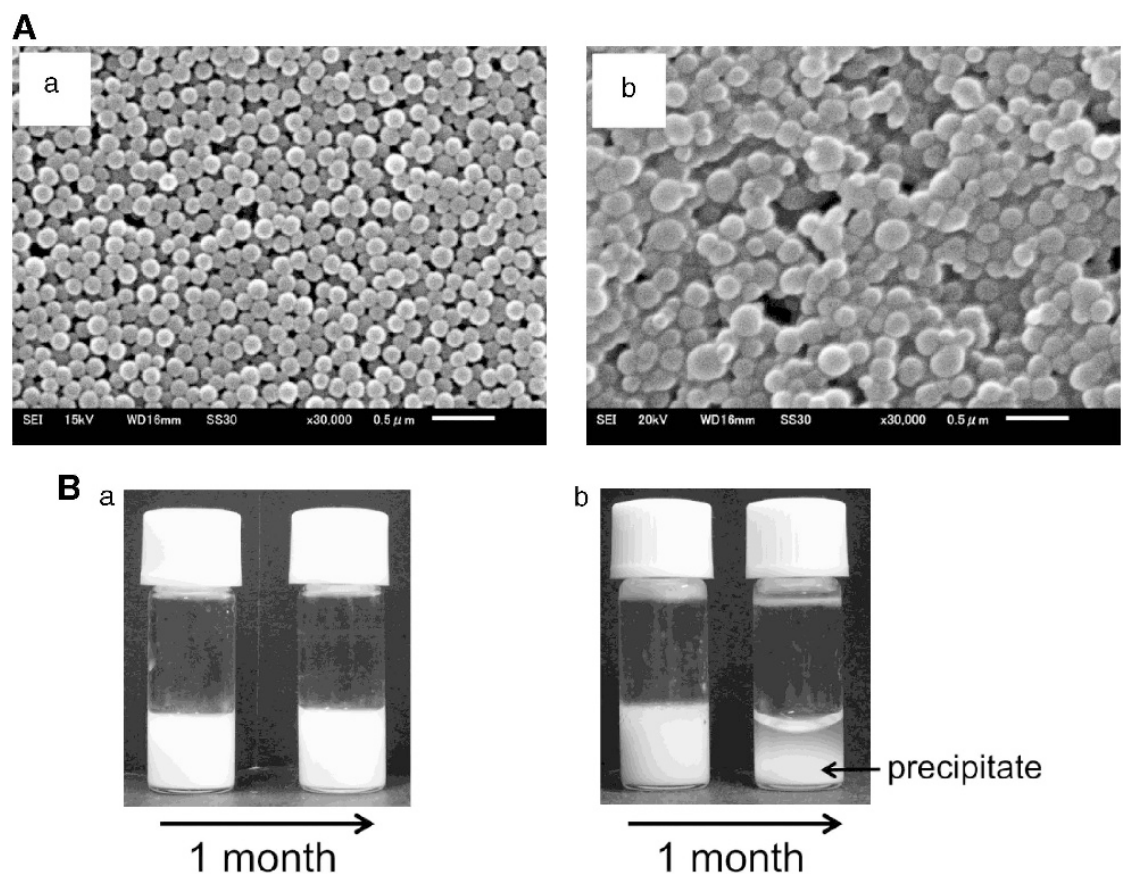

Figure 4 (A) The SEM images of polystyrene particles from the enzymatic emulsifier-free emulsion polymerization prepared using (a) ACAC and (b) CP as the initiator. (B) Photographs of dispersed solutions of particles prepared using (a) ACAC and (b) CP as the initiator. 
located on the surface of the particles, influenced the particle formation and stability. Enzymatic emulsifier-free emulsion polymerization might offer a practical method for preparing polymer particles because polymerizations can be conducted at room temperature using a mild enzymatic process without surfactants. Additional studies that are in progress include improving the reaction efficiency and developing the application of this methodology for other monomers, which will be described in future reports.

\section{ACKNOWLEDGEMENTS}

This work was partly supported by a grant from the Futaba Electronics Memorial Foundation. We thank Professor Jun Matsui of Tohoku University for the dynamic light scattering measurements.

1 Kawaguchi, H. Functional polymer microspheres. Prog. Polym. Sci. 25, 1171-1210 (2000).

2 Arshady, R. Suspension, emulsion, and dispersion polymerization: a methodologica survey. Colloid Polym. Sci. 270, 717-732 (1992).

3 Zetterlund, P. B., Kagawa, Y. \& Okubo, M. Controlled/living radical polymerization in dispersed systems. Chem. Rev. 108, 3747-3794 (2008).

4 Ganachaud, F., Sauzedde, F., Elaïssari, A. \& Pichot, C. Emulsifier-free emulsion copolymerization of styrene with two different amino-containing cationic monomers. I. Kinetic studies. J. Appl. Polym. Sci. 65, 2315-2330 (1997).

5 Kobayashi, S., Uyama, H. \& Kimura, S. Enzymatic polymerization. Chem. Rev. 101, 3793-3818 (2001).

6 Gross, R. A., Kumar, A. \& Kalra, B. Polymer synthesis by in vitro enzyme catalysis. Chem. Rev. 101, 2097-2124 (2001).

7 Kadokawa, J. \& Kobayashi, S. Polymer synthesis by enzymatic catalysis. Curr. Opin. Chem. Biol. 14, 145-153 (2010).

8 Uyama, H. \& Kobayashi, S. Enzymatic synthesis and properties of polymers from polyphenols. Adv. Polym. Sci. 194, 51-67 (2006).
9 Kohri, M., Fukushima, H., Taniguchi, T. \& Nakahira, T. Synthesis of polyarbutin by oxidative polymerization using PEGylated hematin as a biomimetic catalyst. Polym. $J$. 42, 952-955 (2010).

10 Durand, A., Lalot, T., Brigodiot, M. \& Marechal, E. Enzyme-mediated initiation of acrylamide polymerization: reaction mechanism. Polymer 41, 8183-8192 (2000).

11 Singh, A. \& Kaplan, D. L. In vitro enzyme-induced vinyl polymerization. Adv. Polym. Sci. 194, 211-224 (2006).

12 Kurioka, H., Uyama, H. \& Kobayashi, S. Peroxidase-catalyzed dispersion polymerization of phenol derivatives. Polym. J. 30, 526-529 (1998).

13 Uyama, H., Kurioka, H. \& Kobayashi, S. Preparation of polyphenol particles by peroxidase-catalyzed dispersion polymerization. Colloids Surf. A: Physicochem. Eng. Aspects 153, 189-194 (1999).

14 Takamura, S., Takeoka, Y. \& Rikukawa, M. Enzymatic synthesis of poly(aniline) particles. Synth. Metals 135-136, 331-332 (2003).

15 Shan, J., Kitamura, Y. \& Yoshizawa, H. Emulsion polymerization of styrene by horseradish peroxidase-mediated initiation. Colloid Polym. Sci. 284, 108-111 (2005).

16 Qi, G., Jones, C. W. \& Schork, F. J. Enzyme-initiated miniemulsion polymerization Biomacromolecules 7, 2927-2930 (2006).

17 Kohri, M., Kobayashi, A., Fukushima, H., Kojima, T., Taniguchi, T., Saito, K. \& Nakahira, T. Enzymatic miniemulsion polymerization of styrene with a polymerizable surfactant. Polym. Chem. 3, 900-906 (2012).

18 Fukushima, H., Kohri, M., Kojima, T., Taniguchi, T., Saito, K. \& Nakahira, T. Surface-initiated enzymatic vinyl polymerization: synthesis of polymer-grafted silica particles using horseradish peroxidase as catalyst. Polym. Chem. 3, 1123-1125 (2012).

19 Teixeira, D., Lalot, T., Brigodiot, M. \& Marechal, E. $\beta$-Diketones as key compounds in free-radical polymerization by enzyme-mediated initiation. Macromolecules 32, 70-72 (1999).

20 Singh, A., Ma, D. \& Kaplan, D. L. Enzyme-mediated free radical polymerization of styrene. Biomacromolecules 1, 592-596 (2000)

21 Pich, A., Bhattacharya, S. \& Adler, H. -J. P. Composite magnetic particles: 1. Deposition of magnetite by heterocoagulation method. Polymer 46, 1077-1086 (2005).

22 Baader, W. J., Bohne, C., Cilento, G. \& Dunford, H. B. Peroxidase-catalyzed formation of triplet acetone and chemiluminescence from isobutyraldehyde and molecular oxygen. J. Biol. Chem. 260, 10217-10225 (1985).

Supplementary Information accompanies the paper on the Polymer Journal website (http://www.nature.com/pj) 